\title{
Research Article \\ Control and Synchronization of Fractional-Order Financial System Based on Linear Control
}

\author{
Liping Chen, ${ }^{1}$ Yi Chai, ${ }^{1}$ and Ranchao $\mathrm{Wu}^{2}$ \\ ${ }^{1}$ School of Automation, Chongqing University, Chongqing 400030, China \\ 2 School of Mathematics, Anhui University, Hefei 230039, China \\ Correspondence should be addressed to Liping Chen, lip_chen@yahoo.com.cn
}

Received 10 April 2011; Accepted 14 June 2011

Academic Editor: Yong Zhou

Copyright (C) 2011 Liping Chen et al. This is an open access article distributed under the Creative Commons Attribution License, which permits unrestricted use, distribution, and reproduction in any medium, provided the original work is properly cited.

Control and synchronization of the financial systems with fractional-order are discussed in this paper. Based on the stability theory of fractional-order differential equations, Routh-Hurwitz stability condition, and by using linear control, simpler controllers are designed to achieve control and synchronization of the fractional-order financial systems. The proposed controllers are linear and easy to implement, which have improved the existing results. Theoretical analysis and numerical simulations are shown to demonstrate the validity and feasibility of the proposed method.

\section{Introduction}

Chaos, as a very interesting nonlinear phenomenon, has been intensively investigated in many fields over the last four decades. Since the pioneering work [1] that chaotic dynamics could exist in an economical model, research on the dynamical behavior of economical model has become one of the most interesting and important topics which have received increasing attention. Many continuous chaotic models have been proposed to study complex economic dynamics, such as the forced Vander-Pol model [2], the IS-ML model [3], Behrens-Feichtinger model [4], and Cournot-Puu model [5]. Just as all the other chaotic systems in engineering, financial chaotic system has complex dynamical behaviors and possess some special features, such as excessive sensitivity to initial conditions, the complex patterns of phase portraits, positive Lyapunov exponents, and bounded and fractal properties of the motion in the phase space. These features are inherent properties of the system itself, rather than caused by external disturbances, which denote some economic behaviors in the fields of finance, stocks, and social economics. In addition, as a nonlinear system, there exist many attractors with 
different topology, such as fixed points, limit cycles, quasiperiodic attractors, and chaotic attractors in chaotic financial systems, which make the contradiction in economic operation process complex and changeful. Chaotic behavior in financial systems is undesirable due to threatening the safety of investment. Therefore, to study the chaotic behaviors in nonlinear economical systems plays a very important role in decision-making by policy makers. Once the instability of a periodic solution, bifurcation, multiperiodic bifurcation, or similar phenomena appear in the economic chaotic systems, decision makers should take some measures to intervene. Some researches suggest that controlling chaos should improve the performance of a chaotic economy and stabilizing periodic solutions of a chaotic market model may increase economic efficiency [6-10]. In recent years, some authors have paid much attention to chaos control in economic models and obtained some results. For example, Ahmed et al. and Agiza used OGY method to control chaos in economic systems [11, 12], respectively. Pyragas [7] and Holyst and Urbanowicz [9] proposed the delayed feedback control, which has been widely used for controlling chaos in economic models. Sun et al. adopted impulsive control to control a financial model [13]. Du et al. applied phase space compression to control chaos in economic systems [14].

On the other hand, it has been found that many systems in interdisciplinary fields can be described by fractional differential equations, for example, dielectric polarization, electrode-electrolyte polarization, electromagnetic waves, viscoelastic systems, quantitative finance, and diffusion wave [15-17]. Recently, fractional-order systems have become an active research area, particularly in control and synchronization of chaotic systems. Interest has been growing in fractional calculus not only from physicists and engineers but also from researchers in life science and economics [18-20]. In fact, financial variables possessing long memories make fractional models more appropriate for dynamic behaviors in finance. Research on fractional-order financial models has a wider range of applications. In 2008, Chen studied nonlinear dynamics and chaos in a fractional-order financial system [21]. As he pointed out, one of the major differences between fractional-order and integer-order models is that fractional-order models possess memory; that is, the fractional-order model depends on the history of the system. The magnitude of the financial variables such as foreign exchange rates, gross domestic product, interest rates, production, and stock market prices can have very long memory; the reason for describing financial systems using a fractional nonlinear model is that it simultaneously possesses memory and chaos. From then on, researchers set out to investigate the fractional financial models, for instance, in [22] a sliding mode controller was designed for a fractional-order chaotic financial system and in [23] control of a fractional-order chaotic financial system by nonlinear feedback control was discussed. As we know, linear feedback control is especially attractive and has been successfully applied to practical implementation, which was adopted in [24-26] to realize control and synchronization of integer-order chaotic systems. However, there exists substantial difference between fractional-order differential systems and integer-order differential ones. Most of the properties, conclusions, and methods to deal with integer-order systems cannot be simply extended to the case of fractional-order ones. Therefore, results about fractional-order chaotic systems are much less than those of integer-order systems. To the best of our knowledge, there are few results about control and synchronization of fractionalorder systems via linear feedback control [27, 28].

In this paper, based on the stability theory of fractional-order differential equations, Routh-Hurwitz stability condition, we investigate control and synchronization of fractionalorder financial systems proposed by Chen via linear control. Sufficient conditions are established and easy to verify. Compared with sliding mode control and nonlinear feedback 
control used for discussing fractional-order financial systems in [22, 23], the linear control is economic and easy to implement, through which control and synchronization of fractionalorder financial systems will be obtained only by choosing suitable feedback gains. The main job of this paper lies in two aspects. One is to remove chaotic phenomenon from fractional financial system by controlling, which makes prediction impossible in the financial world. The other is to realize harmonious and sustainable development between drive financial systems and response ones by investigating synchronization. The obtained results have a certain value to the theoretical guidance and application.

The remainder of this paper is organized as follows. In Section 2, preliminary results are presented and fractional-order financial system is described. In Section 3, some sufficient criteria for control of the fractional-order financial system are given. In Section 4, we discussed synchronization of the fractional-order financial system via linear error feedback. In Section 5, numerical simulations are given to illustrate the effectiveness of the main results. Finally, conclusions are drawn in Section 6.

\section{Preliminaries and System Description}

Fractional calculus is a generalization of integration and differentiation to a noninteger-order integrodifferential operator $D_{t}^{\alpha}$ defined by

$$
D_{t}^{\alpha}= \begin{cases}\frac{d^{\alpha}}{d t^{\alpha}}, & R(\alpha)>0 \\ 1, & R(\alpha)=0 \\ \int_{a}^{t}(d \tau)^{-\alpha}, & R(\alpha)<0 .\end{cases}
$$

Fractional calculus is being used in various fields gradually, such as biophysics, nonlinear dynamics, informatics, and control engineering [29]. There are some definitions for fractional derivatives. The commonly used definitions are Grunwald-Letnikov (GL), RiemannLiouville (RL), and Caputo (C) definitions.

The Grunwald-Letnikov (GL) derivative with fractional-order $\alpha$ is given by

$$
{ }_{\alpha}^{G} D_{t}^{\alpha} f(t)=\lim _{h \rightarrow 0} f_{h}^{(\alpha)}(t)=\lim _{h \rightarrow 0} h^{-\alpha} \sum_{i=0}^{[(t-\alpha) / h]}(-1)^{i}\left(\begin{array}{l}
\alpha \\
i
\end{array}\right) f(t-i h),
$$

where [-] means the integer part.

The Riemann-Liouvill (RL) fractional derivatives are defined by

$$
{ }_{\alpha}^{R} D_{t}^{\alpha} f(t)=\frac{d^{n}}{d t^{n}} \frac{1}{\Gamma(n-\alpha)} \int_{a}^{t} \frac{f(\tau)}{(t-\tau)^{(\alpha-n+1)}} d \tau, \quad n-1<\alpha<n,
$$

where $\Gamma(\cdot)$ is the gamma function, $\Gamma(\tau)=\int_{0}^{\infty} t^{\tau-1} e^{-t} d t$. 


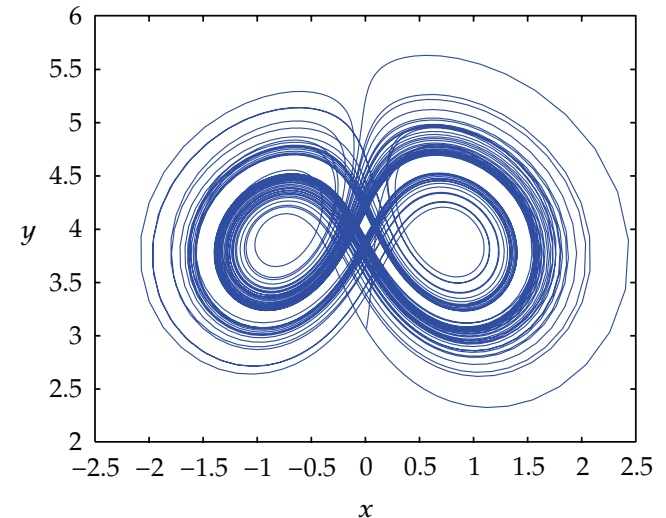

(a)

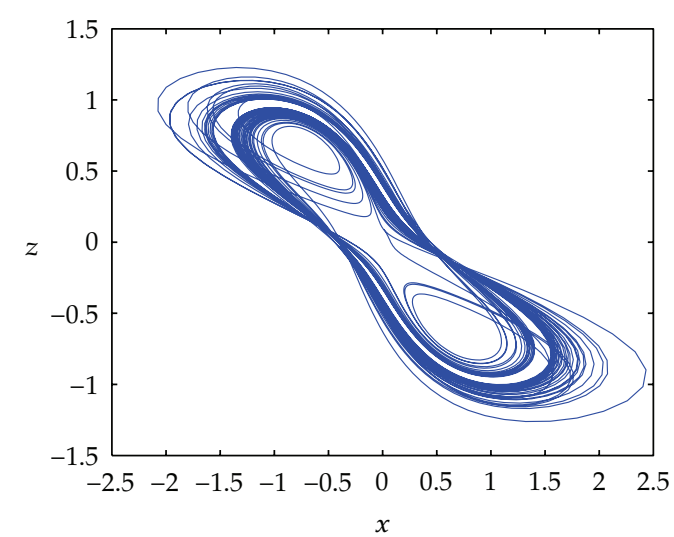

(b)

Figure 1: Chaotic attractors of system (2.5) with order $(0.86,0.92,0.95)$.

The Caputo (C) fractional derivative is defined as follows:

$$
{ }_{\alpha}^{C} D_{t}^{\alpha} f(t)=\frac{1}{\Gamma(n-\alpha)} \int_{a}^{t} f^{(n)}(\tau)(t-\tau)^{(n-\alpha-1)} d \tau, \quad n-1<\alpha<n .
$$

It should be noted that the advantage of Caputo approach is that the initial conditions for fractional differential equations with Caputo derivatives take on the same form as those for integer-order differential, which have well-understood physical meanings. Therefore, in the rest of this paper, the notation $D_{*}^{\alpha}$ is chosen as the Caputo fractional derivative operator ${ }_{\alpha}^{C} D$.

The fractional-order chaotic financial system can be described by

$$
\begin{gathered}
D_{*}^{\alpha_{1}} x=z+(y-a) x, \\
D_{*}^{\alpha_{2}} y=1-b y-x^{2}, \\
D_{*}^{\alpha_{3}} z=-x-c z,
\end{gathered}
$$

where $\alpha_{1}, \alpha_{2}$, and $\alpha_{3}$ are the fractional-order, $\alpha_{1}, \alpha_{2}, \alpha_{3} \in(0,1)$. System (2.5) describes the time variation of three state variables: the interest rate $x$, the investment demand $y$, and the price index $z ; a$ is the saving amount, $b$ is the cost per investment, $c$ is the elasticity of demand of commercial markets, parameters $a, b$, and $c$ are positive real constants. When $a=3, b=0.1$, $c=1$, fractional-order $\left(\alpha_{1}, \alpha_{2}, \alpha_{3}\right)$ is taken as $(0.86,0.92,0.95)$, the largest Lyapunov exponent of system (2.5) is greater than 0 [23]. Figure 1 displays chaotic attractors of system (2.5).

To obtain our results, the following lemma is presented. 
Consider the following fractional-order system:

$$
\begin{aligned}
& D_{*}^{\alpha_{1}} x=f(x, y, z), \\
& D_{*}^{\alpha_{2}} y=g(x, y, z), \\
& D_{*}^{\alpha_{3}} z=h(x, y, z) .
\end{aligned}
$$

Lemma 2.1 (see [30]). System (2.6) is asymptotically stable at the equilibrium points if $\left|\arg \left(\lambda_{i}(A)\right)\right|>\alpha \pi / 2, \alpha=\max \left(\alpha_{1}, \alpha_{2}, \alpha_{3}\right), i=(1,2,3)$, for all eigenvalues $\lambda_{i}$ of the Jacobian matrix $J$ :

$$
J=\left(\begin{array}{lll}
\frac{\partial f}{\partial x} & \frac{\partial f}{\partial y} & \frac{\partial f}{\partial z} \\
\frac{\partial g}{\partial x} & \frac{\partial g}{\partial y} & \frac{\partial g}{\partial z} \\
\frac{\partial h}{\partial x} & \frac{\partial h}{\partial y} & \frac{\partial h}{\partial z}
\end{array}\right)
$$

\section{Chaos Control}

\subsection{Analysis of Equilibrium Points}

Let

$$
\begin{gathered}
z+(y-a) x=0 \\
1-b y-x^{2}=0 \\
-x-c z=0 .
\end{gathered}
$$

It is easy to obtain that system (2.5) has three equilibrium points if

$$
\begin{gathered}
c-b-a b c>0, \\
P_{1}=\left(0, \frac{1}{b}, 0\right), \\
P_{2}=\left(\sqrt{\frac{(c-b-a b c)}{c}}, \frac{(1+a c)}{c},\left(-\frac{1}{c}\right) \cdot \sqrt{\frac{(c-b-a b c)}{c}}\right), \\
P_{3}=\left(-\sqrt{\frac{(c-b-a b c)}{c}}, \frac{(1+a c)}{c},\left(\frac{1}{c}\right) \cdot \sqrt{\frac{(c-b-a b c)}{c}}\right) .
\end{gathered}
$$


The Jacobian matrix of system (2.5) at equilibrium point $\left(x^{*}, y^{*}, z^{*}\right)$ is

$$
J=\left[\begin{array}{ccc}
y^{*}-a & x^{*} & 1 \\
-2 x^{*} & -b & 0 \\
-1 & 0 & -c
\end{array}\right]
$$

The characteristic equation of the Jacobian matrix $J$ is given by

$$
\begin{aligned}
& \lambda^{3}+\left(a+b+c-y^{*}\right) \lambda^{2}+\left(a b+a c+b c-b y^{*}-c y^{*}+2\left(x^{*}\right)^{2}+1\right) \lambda \\
& +a b c-b c y+b+2 c\left(x^{*}\right)^{2}=0 .
\end{aligned}
$$

When $a=3, b=0.1, c=1, P_{1}=(0,10,0)$. Substituting the parameters $a, b$, and $c$ into (3.4), one obtains

$$
\lambda^{3}-5.9 \lambda^{2}-6.6 \lambda-0.6=0
$$

The roots of (3.5) are $\lambda_{1}=6.8730, \lambda_{2}=-0.8730$, and $\lambda_{3}=-0.1$; obviously, $\lambda_{1}$ is a positive real number, based on the lemma, $\arg \left(\lambda_{1}\right)=0, \arg \left(\lambda_{2}\right)=\arg \left(\lambda_{3}\right)=\pi$. So, the equilibrium point $P_{1}$ is unstable.

When $a=3, b=0.1, c=1, P_{2}=(\sqrt{0.6}, 4,-\sqrt{0.6}), P_{3}=(-\sqrt{0.6}, 4, \sqrt{0.6})$. Substituting the parameters $a, b$, and $c$ into (3.4), the characteristic equation of the Jacobian matrix $J$ at equilibrium points $P_{2}, P_{3}$ is

$$
\lambda^{3}+0.1 \lambda^{2}+1.2 \lambda+1.2=0
$$

The roots of (3.6) are $\lambda_{1}=-0.7256, \lambda_{2}=0.3128+1.2474 i$, and $\lambda_{3}=0.3128-1.2474 i$. According to the lemma, $\lambda_{1}$ is a negative real number, $\arg \left(\lambda_{1}\right)=\pi, \arg \left|\left(\lambda_{2,3}\right)\right|=1.3251 . \alpha=2\left|\arg \left(\lambda_{2,3}\right)\right| / \pi=$ 0.8436 . Therefore, we can conclude that if the maximum fractional-order $\alpha$ among $\alpha_{1}, \alpha_{2}, \alpha_{3}$ is less than 0.8436 , the equilibrium points $P_{2}, P_{3}$ are stable. On the contrary, the equilibrium points $P_{2}, P_{3}$ are unstable.

According to the above analysis, when the maximum fractional-order $\alpha$ among $\alpha_{1}, \alpha_{2}$, and $\alpha_{3}$ is less than 0.8436 , there exist two stable equilibrium points; when $\alpha_{1}=\alpha_{2}=\alpha_{3}=$ 0.8436 , system (2.5) will admit a limit cycle; when fractional-order $\alpha_{1}, \alpha_{2}$, and $\alpha_{3}$ are all greater than 0.8436 , there are no stable equilibria, with all the equilibrium points being unstable, which implies that there may exist chaos for system (2.5). By calculating the values of Lyapunov exponents of system (2.5), it could be found that system (2.5) exhibits chaotic behaviors if maximum fractional-order $\alpha$ among $\alpha_{1}, \alpha_{2}$, and $\alpha_{3}$ is greater than or equal to 0.86 [23].

\subsection{Chaos Control}

In this subsection, linear state feedback controller is designed to control fractional-order chaotic financial system to its equilibrium. 
The controlled fractional-order chaotic financial system is given by

$$
\begin{aligned}
& D_{*}^{\alpha_{1}} x=z+(y-a) x+k_{1}\left(x-x^{*}\right) \\
& D_{*}^{\alpha_{2}} y=1-b y-x^{2}+k_{2}\left(y-y^{*}\right) \\
& D_{*}^{\alpha_{3}} z=-x-c z+k_{3}\left(z-z^{*}\right)
\end{aligned}
$$

where $k_{1}, k_{2}$, and $k_{3}$ denote feedback gains and $\left(x^{*}, y^{*}, z^{*}\right)$ is the desired equilibrium point. Obviously, system (3.7) has one equilibrium point $\left(x^{*}, y^{*}, z^{*}\right)$.

The Jacobian matrix of system (3.7) at equilibrium point $\left(x^{*}, y^{*}, z^{*}\right)$ is

$$
J=\left[\begin{array}{ccc}
y^{*}-a+k_{1} & x^{*} & 1 \\
-2 x^{*} & k_{2}-b & 0 \\
-1 & 0 & k_{3}-c
\end{array}\right] .
$$

The characteristic equation of the Jacobian matrix (3.8) is

$$
\begin{aligned}
\lambda^{3} & +\left(a+b+c-k_{1}-k_{2}-k_{3}-y^{*}\right) \lambda^{2} \\
& +\left(a b+a c+b c+k_{1} k_{2}+k_{2} k_{3}+k_{1} k_{3}-(b+c) k_{1}\right. \\
& \left.+(y-a-c) k_{2}-b c y^{*}+(y-a-b) k_{3}+1-b y^{*}+2\left(x^{*}\right)^{2}\right) \lambda \\
& -b c k_{1}+c k_{1} k_{2}+\left(c y^{*}-a c-1\right) k_{2}-\left(b y^{*}+a b+2\left(x^{*}\right)^{2}\right) k_{3}+b k_{1} k_{3} \\
& +(a-y) k_{2} k_{3}-k_{1} k_{2} k_{3}+a b c+b+c-y^{*}+2 c\left(x^{*}\right)^{2}=0 .
\end{aligned}
$$

Our goal is to find suitable feedback gains such that all the state trajectories of system (3.7) are controlled to its equilibrium point, that is to say, roots of (3.9) should satisfy the conditions in the lemma.

Theorem 3.1. When $a=3, b=0.1, c=1, P_{1}=(0,10,0)$; system (3.7) stabilizes to equilibrium point $P_{1}$, if state feedback gains $k_{1}, k_{2}$, and $k_{3}$ satisfy the following conditions:

$$
-10 \leq k_{1}-k_{3}<-6, \quad k_{1}+k_{3}<-6, \quad k_{2}<0.1 .
$$

Proof. Substituting the parameters $a, b$, and $c$ into (3.9), one obtains

$$
\left(10 \lambda+1-10 k_{2}\right)\left(\lambda^{2}-\left(k_{1}+k_{3}+6\right) \lambda+k_{1} k_{3}-k_{1}+7 k_{3}-6\right)=0 .
$$

It is very easy to obtain the roots of (3.11):

$$
\lambda_{1}=k_{2}-0.1, \quad \lambda_{2,3}=\frac{1}{2}\left(k_{1}+k_{3}+6 \pm \sqrt{\left(k_{1}-k_{3}+10\right)\left(k_{1}-k_{3}+6\right)}\right) .
$$


Note that $\lambda_{1}$ is a negative real number, $\lambda_{2,3}$ are a pair of conjugate imaginary roots, and the real parts of imaginary root are negative, that is, $\arg \left(\lambda_{1}\right)=\pi, \arg \left(\lambda_{2,3}\right)>\pi / 2$. Therefore, the trajectory of the controlled fractional-order system (3.7) is asymptotically stable at equilibrium point $P_{1}$.

In Theorem 3.1, we designed three simple linear feedback controllers to ensure controlled system (3.7) stabilized to $P_{1}$. In practice, two linear feedback controllers or single linear feedback controller in controlled system (3.7) will do the same thing. Then we have the following corollaries.

Corollary 3.2. Controlled system (3.7) will approach asymptotically to $P_{1}$ with one of the following conditions about feedback gains:

$$
\begin{gathered}
-10 \leq k_{1}<-6, \quad k_{2}<0.1, \quad k_{3}=0, \\
k_{1}+k_{3}<-6, \quad-10 \leq k_{1}-k_{3}<-6, \quad k_{2}=0 .
\end{gathered}
$$

Corollary 3.3. Controlled system (3.7) will approach asymptotically to $P_{1}$ if feedback gains satisfy

$$
-10 \leq k_{1}<-6, \quad k_{2}=0, \quad k_{3}=0 .
$$

Theorem 3.4. When $a=3, b=0.1, c=1, P_{2}=(\sqrt{0.6}, 4,-\sqrt{0.6})$; if feedback gains $k_{1}, k_{2}$, and $k_{3}$ satisfy one of the following conditions, system (3.7) will approach and stabilizes to equilibrium point $P_{2}$ asymptotically:

$$
\begin{gathered}
k_{1}=0, \quad k_{2}<-0.9, \quad k_{3}=0, \\
k_{1}<\frac{121-\sqrt{62161}}{220}, \quad k_{2}=0, \quad k_{3}=0 .
\end{gathered}
$$

Proof. (1) Substitute the parameters $a=3, b=0.1, c=1$, and $k_{1}=k_{3}=0$ into (3.9), one obtains

$$
\lambda^{3}+\left(\frac{1}{10}-k_{2}\right) \lambda^{2}+\frac{6}{5} \lambda+\frac{6}{5}=0
$$

According to the Routh-Hurwitz criterion, real parts of these eigenvalues $\lambda_{1,2,3}$ of (3.16) are all negative if

$$
\frac{1}{10}-k_{2}>0, \quad\left(\frac{1}{10}-k_{2}\right) \frac{6}{5}>\frac{6}{5}
$$

that is, $k_{2}<-0.9$. That implies that the trajectory of the controlled fractional-order system (3.7) is asymptotically stable at equilibrium point $P_{2}$.

(2) Substituting the parameters $a=3, b=0.1, c=1$, and $k_{2}=k_{3}=0$ into (3.9), one obtains

$$
\lambda^{3}+\left(\frac{1}{10}-k_{1}\right) \lambda^{2}+\left(\frac{6}{5}-\frac{11}{10} k_{1}\right) \lambda+\frac{6}{5}-\frac{1}{10} k_{1}=0 .
$$


By applying the Routh-Hurwitz criterion, if the following conditions for feedback gains are met,

$$
\frac{1}{10}-k_{1}>0, \quad \frac{6}{5}-\frac{11}{10} k_{1}>0, \quad\left(\frac{1}{10}-k_{1}\right)\left(\frac{6}{5}-\frac{11}{10} k_{1}\right)>\frac{6}{5}-\frac{1}{10} k_{1}
$$

then real parts of these eigenvalues $\lambda_{1,2,3}$ are all negative. It follows that $k_{1}<(1 / 220)(121-$ $\sqrt{62161})$. Thus, the trajectory of the controlled fractional-order system (3.7) is asymptotically stable at equilibrium point $P_{2}$.

Remark 3.5. Actually, we adopt single linear feedback controller to stabilize $P_{2}$ of controlled system in Theorem 3.4, namely, stabilizing $P_{2}$ by adding single linear feedback controller on the first state or the second state, but we cannot do it via adding single linear feedback controller on the third state; the reasons are described as follows.

Substituting the parameters $a=3, b=0.1, c=1$, and $k_{1}=k_{2}=0$ into (3.9), one obtains

$$
\lambda^{3}+\left(\frac{1}{10}-k_{3}\right) \lambda^{2}+\left(\frac{6}{5}+\frac{9}{10} k_{3}\right) \lambda-\frac{11}{10} k_{3}+\frac{6}{5}=0
$$

By using the Routh-Hurwitz criterion, real parts of these eigenvalues $\lambda_{1,2,3}$ are all negative if

$$
\frac{1}{10}-k_{3}>0, \quad-\frac{11}{10} k_{3}+\frac{6}{5}>0, \quad\left(\frac{1}{10}-k_{3}\right)\left(\frac{6}{5}+\frac{9}{10} k_{3}\right)>-\frac{11}{10} k_{3}+\frac{6}{5}
$$

By using simple calculation, such $k_{3}$ is obviously absent. So the trajectory of the controlled fractional-order system (3.7) is not asymptotically stable at equilibrium point $P_{2}$.

Theorem 3.6. When $a=3, b=0.1, c=1, P_{2}=(-\sqrt{0.6}, 4, \sqrt{0.6})$; if feedback gains $k_{1}, k_{2}$, and $k_{3}$ satisfy one of the following conditions, system (3.7) will approach and stabilize to equilibrium point $P_{2}$ asymptotically:

$$
\begin{gathered}
k_{1}=0, \quad k_{2}<-0.9, \quad k_{3}=0, \\
k_{1}<\frac{121-\sqrt{62161}}{220}, \quad k_{2}=0, \quad k_{3}=0 .
\end{gathered}
$$

Proof. The proof is the same as that of Theorem 3.4 and so we omit it here.

\section{Chaos Synchronization}

In this section, we will investigate synchronization of fractional-order financial system (2.5). Three and two simple linear feedback controllers are designed to achieve synchronization, 
which simplify the existing synchronization schemes and reduce the synchronization cost. Drive system and response system are described as follows, respectively:

$$
\begin{aligned}
& D_{*}^{\alpha_{1}} x_{m}=z_{m}+\left(y_{m}-a\right) x_{m}, \\
& D_{*}^{\alpha_{2}} y_{m}=1-b y_{m}-x_{m}^{2}, \\
& D_{*}^{\alpha_{3}} z_{m}=-x_{m}-c z_{m}, \\
& D_{*}^{\alpha_{1}} x_{s}=z_{s}+\left(y_{s}-a\right) x_{s}+u_{1}, \\
& D_{*}^{\alpha_{2}} y_{s}=1-b y_{s}-x_{s}^{2}+u_{2}, \\
& D_{*}^{\alpha_{3}} z_{s}=-x_{s}-c z_{s}+u_{3},
\end{aligned}
$$

where $u_{1}, u_{2}$, and $u_{3}$ denote the external control inputs, to be designed later. It follows from systems (4.1) and (4.2) that the following error dynamical system is

$$
\begin{aligned}
& D_{*}^{q_{1}} e_{1}=\left(y_{m}-a\right) e_{1}+x_{s} e_{2}+e_{3}-u_{1}, \\
& D_{*}^{q_{2}} e_{2}=\left(x_{m}+x_{s}\right) e_{1}-b e_{2}-u_{2}, \\
& D_{*}^{q_{3}} e_{3}=-e_{1}-c e_{3}-u_{3},
\end{aligned}
$$

where $e_{1}=x_{m}-x_{s}, e_{2}=y_{m}-y_{s}$, and $e_{3}=z_{m}-z_{s}$. Our aim is to find suitable control laws $u_{i}(i=1,2,3)$ for stabilizing the error dynamics system (4.3). To this end, the following theorem is proposed.

Theorem 4.1. For any given initial conditions, synchronization between systems (4.1) and (4.2) will occur if control schemes are defined as follows:

$$
\begin{aligned}
& u_{1}=k_{1} e_{1}, \\
& u_{2}=k_{2} e_{2}, \\
& u_{3}=k_{3} e_{3},
\end{aligned}
$$

where $k_{1}, k_{2}$, and $k_{3}$ are feedback gains and satisfy the following conditions:

$$
\begin{aligned}
& k_{1}>L-a, \\
& k_{2}<-b-\frac{9 L^{2}}{4\left(L-a-k_{1}\right)}, \\
& k_{3}<-c .
\end{aligned}
$$


Proof. Combining (4.3) with (4.4), the error system (4.3) is given by

$$
\begin{aligned}
& D_{*}^{q_{1}} e_{1}=\left(y_{m}-a-k_{1}\right) e_{1}+x_{s} e_{2}+e_{3}, \\
& D_{*}^{q_{2}} e_{2}=\left(x_{m}+x_{s}\right) e_{1}-\left(b+k_{2}\right) e_{2}, \\
& D_{*}^{q_{3}} e_{3}=-e_{1}-\left(c+k_{3}\right) e_{3} .
\end{aligned}
$$

Error dynamical system (4.6) can be rewritten as the following matrix form:

$$
\left[\begin{array}{c}
D_{*}^{q_{1}} e_{1} \\
D_{*}^{q_{2}} e_{2} \\
D_{*}^{q_{3}} e_{3}
\end{array}\right]=A\left[\begin{array}{l}
e_{1} \\
e_{2} \\
e_{3}
\end{array}\right]
$$

where

$$
A=\left[\begin{array}{ccc}
y_{m}-a-k_{1} & x_{s} & 1 \\
x_{m}+x_{s} & -\left(b+k_{2}\right) & 0 \\
-1 & 0 & -\left(c+k_{3}\right)
\end{array}\right] .
$$

Suppose that $\lambda$ is one of the eigenvalues of matrix $A$ and the corresponding noneigenvector is $\varepsilon=\left(\varepsilon_{1}, \varepsilon_{2}, \varepsilon_{3}\right)^{T}$, that is,

$$
A \varepsilon=\lambda \varepsilon
$$

Taking conjugate transposal on both sides of (4.9), one obtains

$$
(A \varepsilon)^{H}=\bar{\lambda} \varepsilon^{H} .
$$

Equation (4.9) multiplied left by $1 / 2 \varepsilon^{H}$ plus (4.10) multiplied right by $1 / 2 \varepsilon$, we have

$$
\varepsilon^{H}\left(\frac{1}{2} A+\frac{1}{2} A^{H}\right) \varepsilon=\frac{1}{2}(\lambda+\bar{\lambda}) \varepsilon^{H} \varepsilon
$$


Because a chaotic system has bounded trajectories, there exists a positive constant $L$, such that $|x|<L,|y|<L$. Thus,

$$
\begin{aligned}
\varepsilon^{H} \frac{1}{2}\left(A+A^{H}\right) \varepsilon & =\left[\varepsilon_{1}^{H}, \varepsilon_{2}^{H}, \varepsilon_{3}^{H}\right]\left[\begin{array}{ccc}
y_{m}-a-k_{1} & \frac{x_{m}+2 x_{s}}{2} & 0 \\
\frac{x_{m}+2 x_{s}}{2} & -\left(b+k_{2}\right) & 0 \\
0 & 0 & -\left(c+k_{3}\right)
\end{array}\right]\left[\begin{array}{l}
\varepsilon_{1} \\
\varepsilon_{2} \\
\varepsilon_{3}
\end{array}\right] \\
& \leq\left[\left|\varepsilon_{1}^{H}\right|,\left|\varepsilon_{2}^{H}\right|,\left|\varepsilon_{3}^{H}\right|\right]\left[\begin{array}{ccc}
L-a-k_{1} & \frac{3}{2} L & 0 \\
\frac{3}{2} L & -\left(b+k_{2}\right) & 0 \\
0 & 0 & -\left(c+k_{3}\right)
\end{array}\right]\left[\begin{array}{l}
\left|\varepsilon_{1}\right| \\
\left|\varepsilon_{2}\right| \\
\left|\varepsilon_{3}\right|
\end{array}\right] .
\end{aligned}
$$

From (4.11), we have

$$
\begin{aligned}
\frac{1}{2}(\lambda+\bar{\lambda}) & =\frac{\varepsilon^{H}\left((1 / 2) A+(1 / 2) A^{H}\right) \varepsilon}{\varepsilon^{H} \varepsilon} \\
& \leq \frac{1}{\varepsilon^{H} \varepsilon}\left[\left|\varepsilon_{1}^{H}\right|,\left|\varepsilon_{2}^{H}\right|,\left|\varepsilon_{3}^{H}\right|\right] P\left[\left|\varepsilon_{1}\right|,\left|\varepsilon_{2}\right|,\left|\varepsilon_{3}\right|\right]^{T},
\end{aligned}
$$

where

$$
P=\left[\begin{array}{ccc}
L-a-k_{1} & \frac{3}{2} L & 0 \\
\frac{3}{2} L & -\left(b+k_{2}\right) & 0 \\
0 & 0 & -\left(c+k_{3}\right)
\end{array}\right]
$$

It is obvious that real parts of all eigenvalues $\lambda$ are negative and matrix $P$ should be negative definite, namely, the following inequalities hold:

$$
\begin{gathered}
L-a-k_{1}<0, \\
-\left(b+k_{2}\right)\left(L-a-k_{1}\right)-\frac{9}{4} L^{2}<0, \\
\left(b+k_{2}\right)\left(c+k_{3}\right)\left(L-a-k_{1}\right)+\frac{9}{4} L^{2}\left(c+k_{3}\right)<0 .
\end{gathered}
$$

Simplifing the above inequalities, one has

$$
\begin{aligned}
& k_{1}>L-a, \\
& k_{2}<-b-\frac{9 L^{2}}{4\left(L-a-k_{1}\right)}, \\
& k_{3}<-c .
\end{aligned}
$$


Therefore, based on stability theorem of fractional-order systems, error system (4.6) is asymptotically stable at the origin, which implies that synchronization between systems (4.1) and (4.2) will be achieved.

Based on the above analysis, it is easy to obtain that two linear feedback controllers could also achieve synchronization between systems (4.1) and (4.2). Then, we have the following corollary.

Corollary 4.2. For any given initial condition, if control schemes are described as $u_{1}=k_{1} e_{1}, u_{3}=$ $k_{3} e_{3}$ and feedback gains satisfy

$$
L-a<k_{1}<L-a+\frac{9}{4 b} L^{2}, \quad k_{3}<-c,
$$

then the response system (4.2) can synchronize the drive system (4.1).

Proof. The proof is similar to that of Theorem 4.1. After some computations, we have

$$
\begin{aligned}
\frac{1}{2}(\lambda+\bar{\lambda}) & =\frac{\varepsilon^{H}\left((1 / 2) A+1 / 2 A^{H}\right) \varepsilon}{\varepsilon^{H} \mathcal{E}} \\
& \leq \frac{1}{\varepsilon^{H} \varepsilon}\left[\left|\varepsilon_{1}^{H}\right|,\left|\varepsilon_{2}^{H}\right|,\left|\varepsilon_{3}^{H}\right|\right] P\left[\left|\varepsilon_{1}\right|,\left|\varepsilon_{2}\right|,\left|\varepsilon_{3}\right|\right]^{T},
\end{aligned}
$$

where

$$
P=\left[\begin{array}{ccc}
L-a-k_{1} & \frac{3}{2} L & 0 \\
\frac{3}{2} L & -b & 0 \\
0 & 0 & -\left(c+k_{3}\right)
\end{array}\right]
$$

Matrix $P$ must be negative definite; if the following inequalities hold:

$$
\begin{gathered}
L-a-k_{1}<0, \\
-b\left(L-a-k_{1}\right)-\frac{9}{4} L^{2}<0, \\
b\left(c+k_{3}\right)\left(L-a-k_{1}\right)+\frac{9}{4} L^{2}\left(c+k_{3}\right)<0,
\end{gathered}
$$

then one obtains

$$
L-a<k_{1}<L-a+\frac{9}{4 b} L^{2}, \quad k_{3}<-c .
$$

Therefore, real parts of all eigenvalues $\lambda$ are negative; according to the stability theorem of fractional-order systems, error system (4.6) is asymptotically stable. This means that the slave system (4.2) can asymptotically synchronize the master system (4.1). 

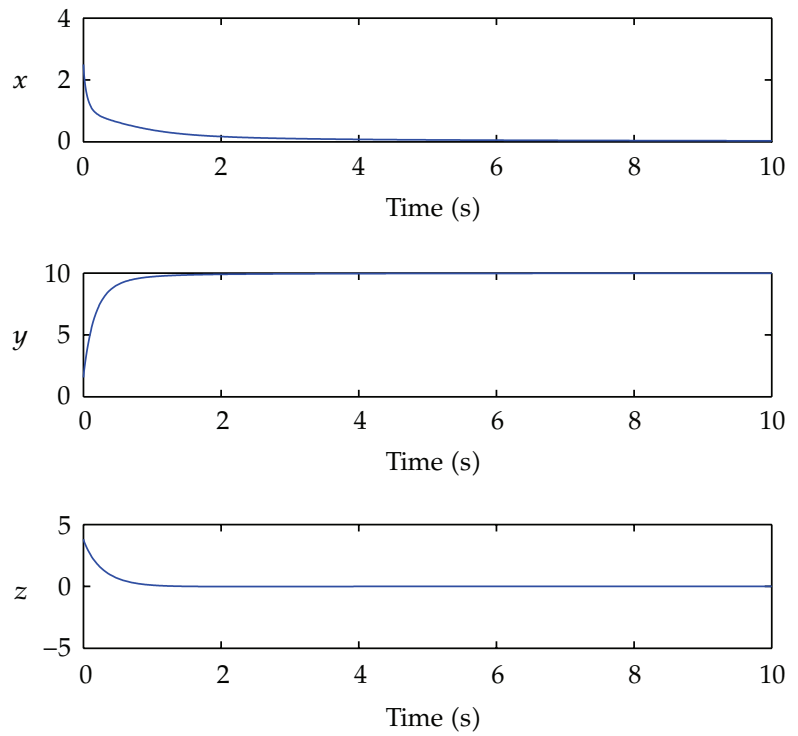

Figure 2: The time response of the states for stabilizing system (3.7) to $P_{1}\left(k_{1}=-9, k_{2}=-5, k_{3}=-2\right)$.

Remark 4.3. References $[22,23]$ presented control of the model by using sliding mode control and nonlinear control, respectively, but with no consideration of synchronization. In contrast, control cost of linear control is low.

Remark 4.4. Response time of control and synchronization could be adjusted with suitable state feedback gains and error feedback gains in allowed limits.

\section{Numerical Simulations}

In this section, to verify theoretical results obtained in the previous section, the corresponding numerical simulations will be performed and an improved predictor-corrector algorithm is applied (see the appendix). In all simulations, fractional-order $\left(\alpha_{1}, \alpha_{2}\right.$, and $\left.\alpha_{3}\right)$ is chosen as $(0.86,0.92$, and 0.95$)$ to ensure the existence of chaos in system (2.5).

\section{Chaos Control}

The parameters of system (2.5) are selected as $a=3, b=0.1$, and $c=1$. The initial values of controlled system (3.7) are chosen as $(x(0), y(0), z(0))=(3,1,4)$, respectively. Based on Theorem 3.1, we chose state feedback gain $\left(k_{1}, k_{2}, k_{3}\right)$ as $(-9,-5,-2)$, under these conditions, roots of (3.11) are $\lambda_{1}=-5.1, \lambda_{1}=-2.5+0.866 i$, and $\lambda_{1}=-2.5-0.866 i$. Controlled system (3.7) is asymptotically stable at $P_{1}$. Figure 2 shows that the controlled system (3.7) can be stabilized to $P_{1}$. When state feedback gain $\left(k_{1}, k_{2}, k_{3}\right)=(0,-3,0)$ and $(-2,0,0)$, roots $\left(\lambda_{1}, \lambda_{2}, \lambda_{3}\right)$ of $(3.16)$ and $(3.18)$ are $(-2.8256,-0.1372-0.6371 i,-0.1372+0.6371 i)$ and $(-2.8256,-0.1372+0.6371 i,-0.1372-0.6371 i)$, respectively. According to Theorem 3.4, controlled system (3.7) will stabilize at $P_{2}$. Figures 3 and 4 display the simulation results, respectively. When the parameters of the controlled system (3.7) are selected as above, based on Theorem 3.6, $P_{3}$ is stabilized. Figures 5 and 6 display the stabilization of the equilibrium point $P_{3}$ for state feedback gain $(0,-8,0)$ and $(-2,0,0)$, respectively. 

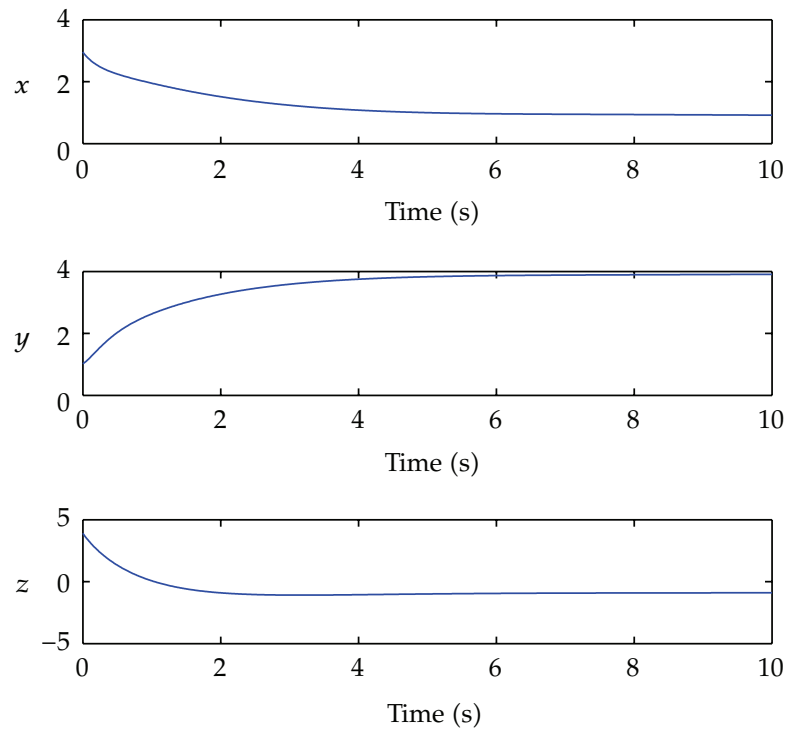

Figure 3: The time response of the states for stabilizing system (3.7) to $P_{2}\left(k_{1}=0, k_{2}=-3, k_{3}=0\right)$.
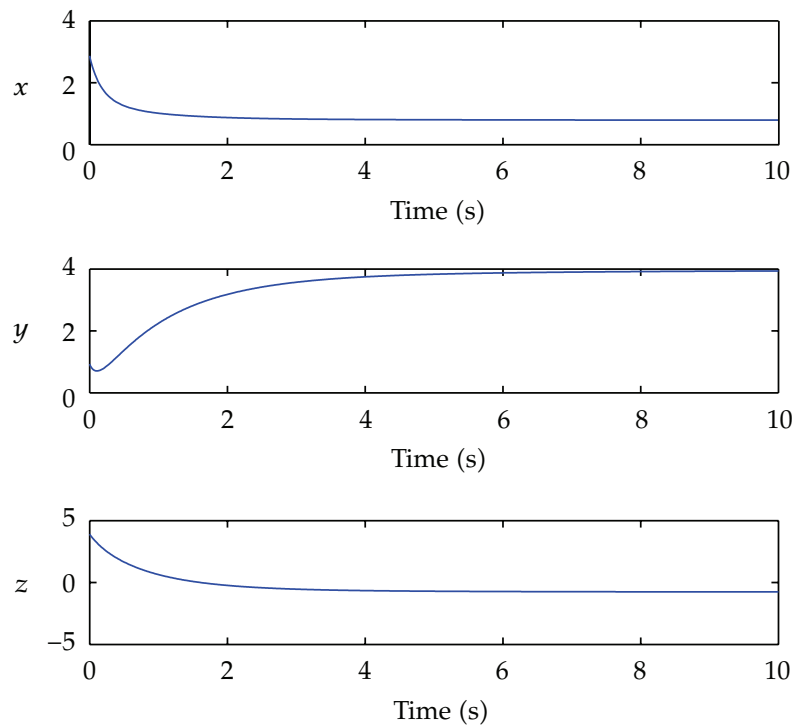

Figure 4: The time response of the states for stabilizing system (3.7) to $P_{2}\left(k_{1}=-2, k_{2}=0, k_{3}=0\right)$.

\section{Chaos Synchronization}

When the parameters of system (2.5) are chosen as $a=3, b=0.1$, and $c=1$, select the initial values of the drive and the response systems as $\left(x_{m}(0), y_{m}(0), z_{m}(0)\right)=(4,-3,2)$ and $\left(x_{s}(0), y_{s}(0), z_{s}(0)\right)=(-3,2,4)$, respectively. For error feedback gain $\left(k_{1}, k_{2}, k_{3}\right)=(12,9,-2)$, simulation result of the synchronization between systems (4.1) and (4.2) is shown in Figure 7. The synchronization error states between systems (4.1) and (4.2) and displayed in Figure 8. When error feedback gains $k_{1}=8, k_{2}=0$, and $k_{3}=-2$, it can be seen that the derive 

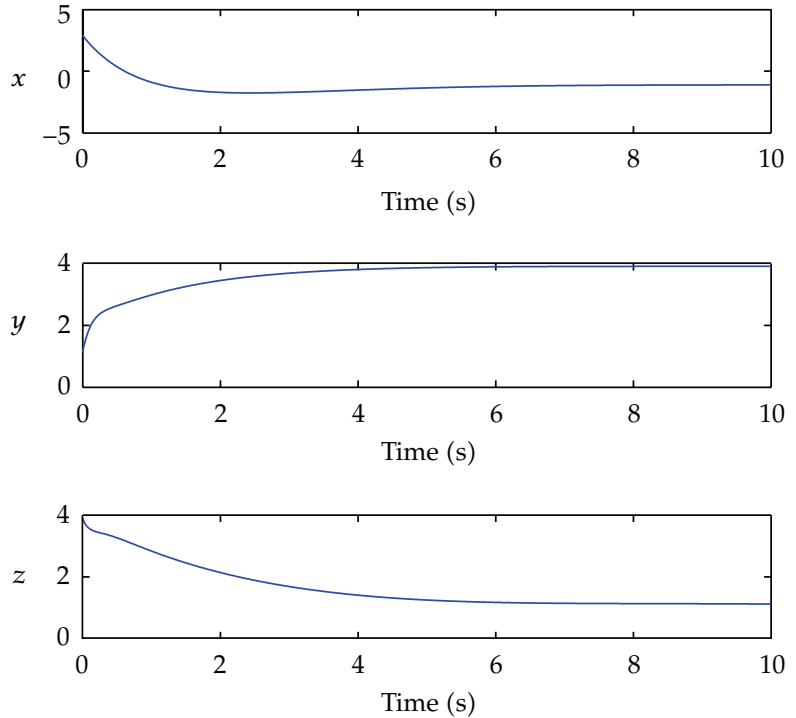

Figure 5: The time response of the states for stabilizing system (3.7) to $P_{3}\left(k_{1}=0, k_{2}=-8, k_{3}=0\right)$.
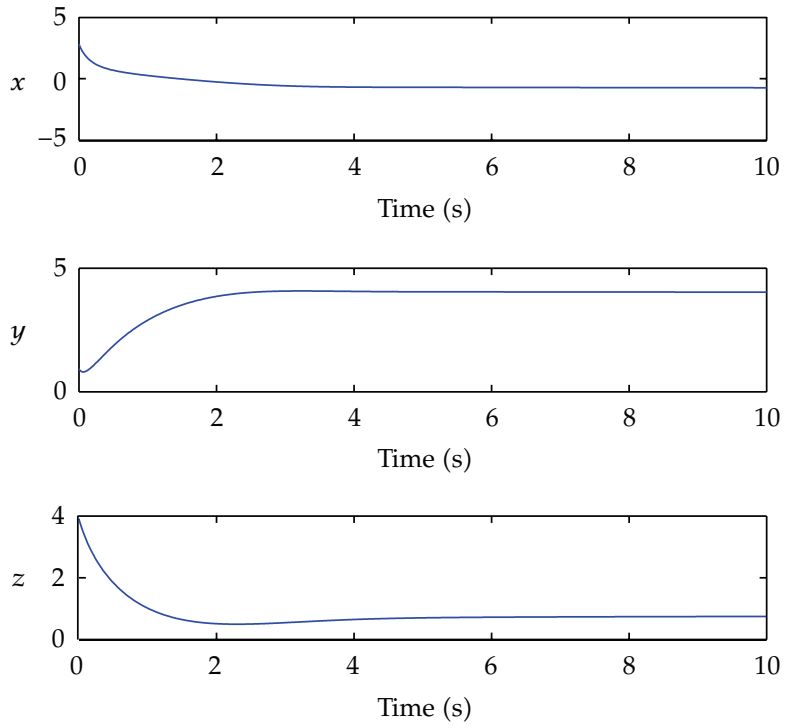

Figure 6: The time response of the states for stabilizing system (3.7) to $P_{3}\left(k_{1}=-2, k_{2}=0, k_{3}=0\right)$.

system (4.1) and the response system (4.2) achieve the synchronization in Figure 9. Figure 10 displays the error state time response between systems (4.1) and (4.2).

\section{Conclusion}

In this paper, based on the stability theory of fractional-order systems and Routh-Hurwitz stability condition, some sufficient conditions for control and synchronization of the fractional-order chaotic financial system by linear feedback control have been derived. Finally, numerical simulations are provided to verify the effectiveness of the results obtained. The results 

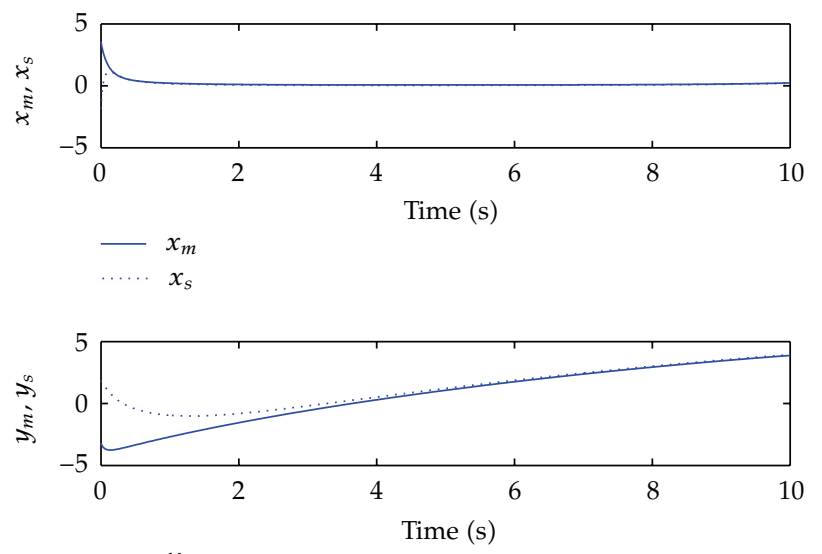

$-y_{m}$

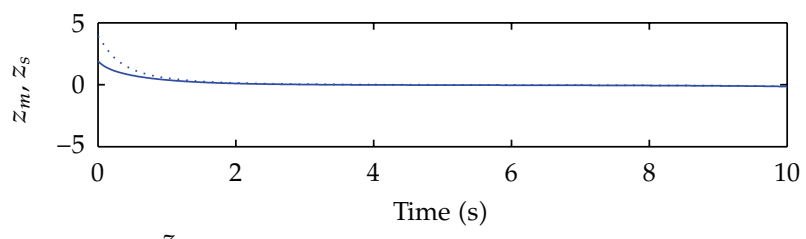

$z_{m}$

$z_{S}$

Figure 7: Synchronization states of systems (4.1) and (4.2) $\left(k_{1}=12, k_{2}=9, k_{3}=-2\right)$.
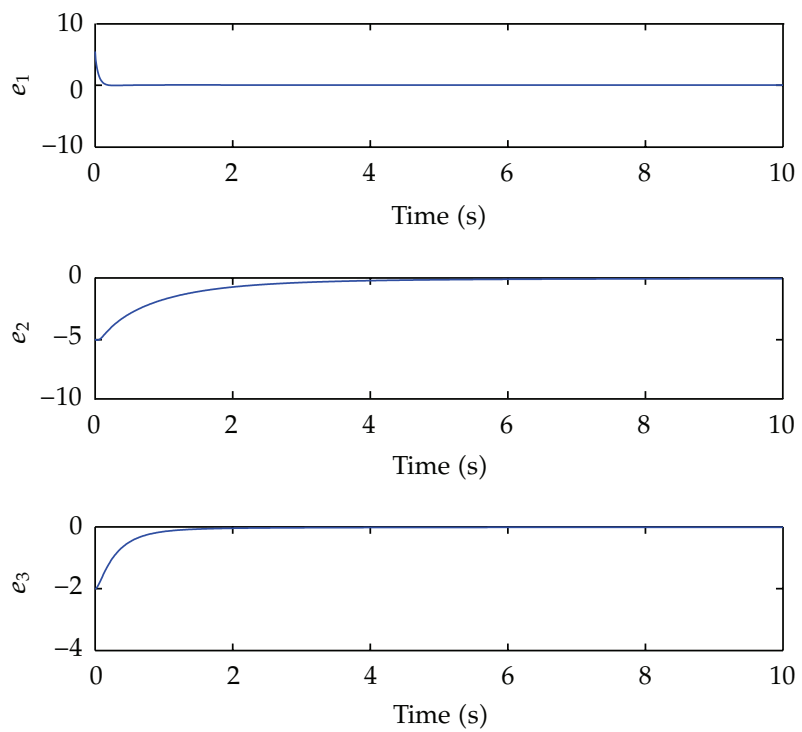

Figure 8: Synchronization error states of systems (4.1) and (4.2) $\left(k_{1}=12, k_{2}=9, k_{3}=-2\right)$. 

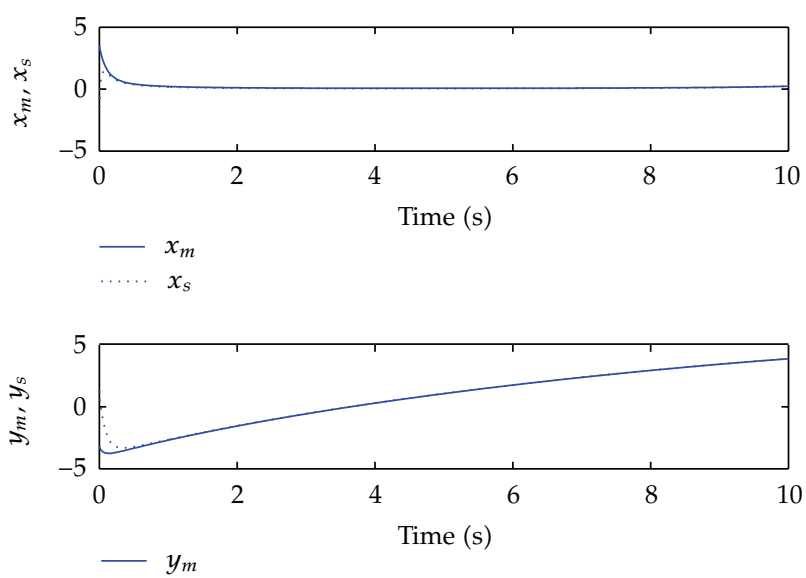

$y_{s}$

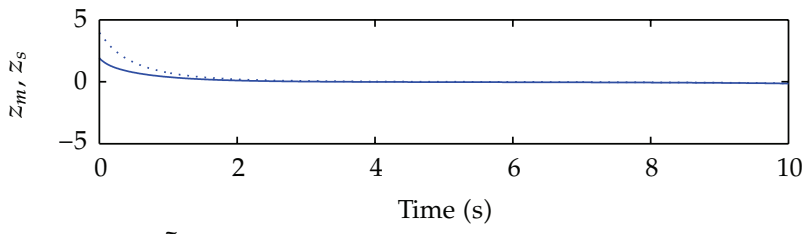

$z_{m}$

Figure 9: Synchronization states of systems (4.1) and (4.2) $\left(k_{1}=8, k_{2}=0, k_{3}=-2\right)$.

are economical, reliable and efficient. It is noted that the method applied in the paper can also be extended to other fractional-order chaotic systems.

\section{Appendix}

An improved predictor-corrector algorithm [31] for fractional-order differential equations is presented in brief. In comparison with the classical one-step Adams-Bashforth-Moulton algorithm, the numerical approximation of the improved algorithm is more accurate and the computational cost is lower.

The following differential equation:

$$
\begin{gathered}
\frac{d^{\alpha} x}{d t^{\alpha}}=f(t, x), \quad 0 \leq t \leq T, \\
x^{(k)}=x_{0}^{(k)}, \quad k=0,1,2, \ldots,[\alpha]-1,
\end{gathered}
$$

is equivalent to the Volterra integral equation [32]

$$
x=\sum_{k=0}^{[\alpha]-1} x_{0}^{(k)} \frac{t^{k}}{k !}+\frac{1}{\Gamma(\alpha)} \int_{0}^{t} \frac{f(\tau, x)}{(t-\tau)^{1-\alpha}} d \tau .
$$



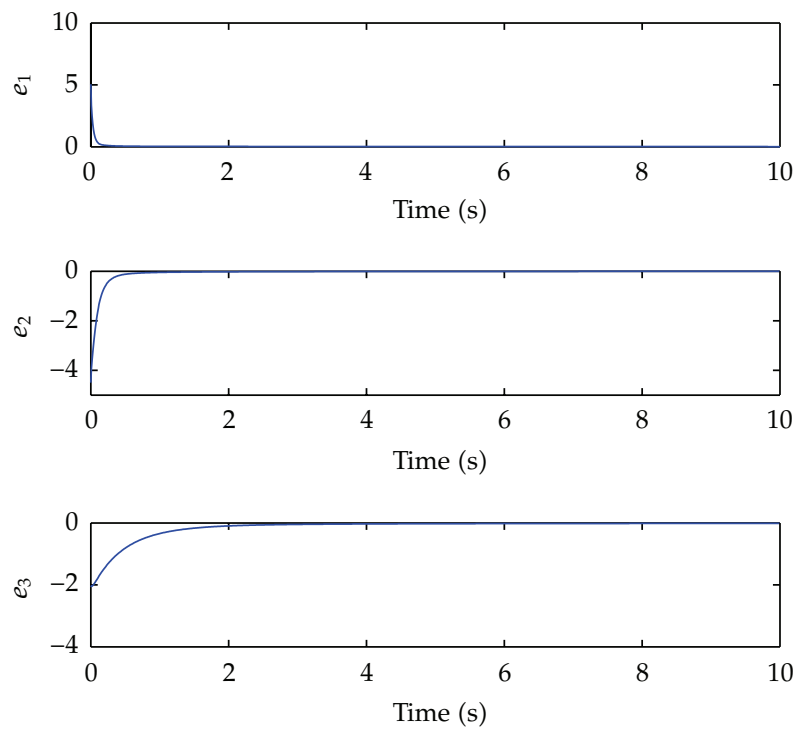

Figure 10: Synchronization error states of systems (4.1) and (4.2) $\left(k_{1}=8, k_{2}=0, k_{3}=-2\right)$.

Set $h=T / N, t_{n}=n h(n=0,1,2, \ldots, N)$. Then the above equation can be discretized as follows:

$$
x_{h}\left(t_{n+1}\right)=\sum_{k=0}^{[\alpha]-1} x_{0}^{(k)} \frac{t^{k}}{k !}+\frac{h^{\alpha}}{\Gamma(\alpha+2)} f\left(t_{n+1}, x_{h}^{\rho}\left(t_{n+1}\right)\right)+\frac{h^{\alpha}}{\Gamma(\alpha+2)} \sum_{j=0}^{n} a_{j, n+1} f\left(t_{j}, x_{h}\left(t_{j}\right)\right),
$$

where

$$
\begin{gathered}
a_{j, n+1}= \begin{cases}n^{\alpha+1}-(n-\alpha)(n+1)^{\alpha+1}, & j=0, \\
(n-j+2)^{\alpha+1}+(n-j)^{\alpha+1}-2(n-j+1)^{a+1}, & 1 \leq j \leq n, \\
1, & j=n+1,\end{cases} \\
x_{h}^{\rho}\left(t_{n+1}\right)=\sum_{k=0}^{[\alpha]-1} x_{0}^{(k)} \frac{t_{n+1}^{k}}{k !}+\frac{1}{\Gamma(\alpha)} \sum_{j=0}^{n} b_{j, n+1} f\left(t_{j}, x_{h}\left(t_{j}\right)\right),
\end{gathered}
$$

in which, $b_{j, n+1}=h^{\alpha} / \alpha\left((n-j+1)^{\alpha}-(n-j)^{\alpha}\right)$.

The error estimate $e$ is $\operatorname{Max}\left|x\left(t_{j}\right)-x_{h}\left(t_{j}\right)\right|=O\left(h^{\rho}\right)(j=0,1, \ldots, N)$, where $\rho=\operatorname{Min}(2,1+$ $\alpha)$.

\section{Acknowledgments}

The authors would thank the referee and the editor for their valuable comments and suggestions. This work is supported by the National Natural Science Foundation of China (no. 60974090), the Fundamental Research Funds for the Central Universities (no. 
CDJXS11172237), the Specialized Research Fund for the Doctoral Program of Higher Education of China (no. 20093401120001; no. 102063720090013), the Natural Science Foundation of Anhui Province (no. 11040606M12), and the Natural Science Foundation of Anhui Education Bureau (no. KJ2010A035).

\section{References}

[1] R. H. Strotz, J. C. Mcanulty, and J. B. Naines, “Goodwin's nonlinear theory of the business cycle: an electro-analog solution," Econometric, vol. 21, no. 3, pp. 390-411, 1953.

[2] A. C. Chian, "Nonlinear dynamics and chaos in macroeconomics," International Journal of Theoretical and Applied Finance, vol. 3, no. 3, p. 601, 2000.

[3] L. Fanti and P. Manfredi, "Chaotic business cycles and fiscal policy: an IS-LM model with distributed tax collection lags," Chaos, Solitons E Fractals, vol. 32, no. 2, pp. 736-744, 2007.

[4] G. Feichtinger, Economic Evolution and Demographic Change, Springer, Berlin, Germany, 1992.

[5] T. Puu, "Chaos in duopoly pricing," Chaos, Solitons \& Fractals, vol. 1, no. 6, pp. 573-581, 1991.

[6] M. Kopel, "Improving the performance of an economic system: controlling chaos," Journal of Evolutionary Economics, vol. 7, no. 3, pp. 269-289, 1997.

[7] K. Pyragas, "Continuous control of chaos by self-controlling feedback," Physics Letters A, vol. 170, no. 6, pp. 421-428, 1992.

[8] J. A. Holyst, T. Hagel, G. Haag, and W. Weidlich, "How to control a chaotic economy?" Journal of Evolutionary Economics, vol. 6, no. 1, pp. 31-42, 1996.

[9] J. A. Holyst and K. Urbanowicz, "Chaos control in economical model by time-delayed feedback method," Physica A, vol. 287, no. 3-4, pp. 587-598, 2000.

[10] L. Chen and G. Chen, "Controlling chaos in an economic model," Physica A, vol. 374, no. 1, pp. 349358, 2007.

[11] E. Ahmed, A. El-Misiery, and H. N. Agiza, "On controlling chaos in an inflation-unemployment dynamical system," Chaos, Solitons E Fractals, vol. 10, no. 9, pp. 1567-1570, 1999.

[12] H. N. Agiza, "On the analysis of stability, bifurcation, chaos and chaos control of Kopel map," Chaos, Solitons E Fractals, vol. 10, no. 11, pp. 1909-1916, 1999.

[13] J. T. Sun, F. Qiao, and Q. Wu, "Impulsive control of a financial model," Physics Letters A, vol. 335, no. 4, pp. 282-288, 2005.

[14] J. G. Du, T. W. Huang, Z. H. Sheng, and H. B. Zhang, "A new method to control chaos in an economic system," Applied Mathematics and Computation, vol. 217, no. 6, pp. 2370-2380, 2010.

[15] R. C. Koeller, "Polynomial operators, stieltjes convolution, and fractional calculus in hereditary mechanics," Acta Mechanica, vol. 58, no. 3-4, pp. 251-264, 1986.

[16] R. C. Koeller, "Applications of fractional calculus to the theory of viscoelasticity," Journal of Applied Mechanics, vol. 51, no. 2, pp. 294-298, 1984.

[17] O. Heaviside, Electromagnetic Theory, Chelsea, New York, NY, USA, 1971.

[18] Á. Cartea and D. del-Castillo-Negrete, "Fractional diffusion models of option prices in markets with jumps," Physica A, vol. 374, no. 2, pp. 749-763, 2007.

[19] N. Laskin, "Fractional market dynamics," Physica A, vol. 287, no. 3-4, pp. 482-492, 2000.

[20] E. Scalas, R. Gorenflo, and F. Mainardi, "Fractional calculus and continuous-time finance," Physica A, vol. 284, no. 1-4, pp. 376-384, 2000.

[21] W. C. Chen, "Nonlinear dynamics and chaos in a fractional-order financial system," Chaos, Solitons $\mathcal{E}$ Fractals, vol. 36, no. 5, pp. 1305-1314, 2008.

[22] S. Dadras and H. R. Momeni, "Control of a fractional-order economical system via sliding mode," Physica A, vol. 389, no. 12, pp. 2434-2442, 2010.

[23] M. S. Abd-Elouahab, N. EddineHamri, and J. W. Wang, "Chaos control of a fractional-order financial system," Mathematical Problems in Engineering, vol. 2010, Article ID 270646, 18 pages, 2010.

[24] Y. Chen, X. Wu, and Z. Gui, "Global synchronization criteria for a class of third-order non-autonomous chaotic systems via linear state error feedback control," Applied Mathematical Modelling, vol. 34, no. 12, pp. 4161-4170, 2010.

[25] J. X. Zhang and W. S. Tang, "Control and synchronization for a class of new chaotic systems via linear feedback," Nonlinear Dynamics, vol. 58, no. 4, pp. 675-686, 2009.

[26] M. Sun, Q. Jia, and L. Tian, "A new four-dimensional energy resources system and its linear feedback control," Chaos, Solitons \& Fractals, vol. 39, no. 1, pp. 101-108, 2009. 
[27] B. G. Xin, T. Chen, and Y. Q. Liu, "Synchronization of chaotic fractional-order windmt systems via linear state error feedback control," Mathematical Problems in Engineering, Article ID 859685, 10 pages, 2010.

[28] Z. M. Odibat, N. Corson, M. A. Aziz-Alaoui, and C. Bertelle, "Synchronization of chaotic fractionalorder systems via linear control," International Journal of Bifurcation and Chaos in Applied Sciences and Engineering, vol. 20, no. 1, pp. 81-97, 2010.

[29] R. Hilfer, Applications of Fractional Calculus in Physics, World Scientific, Singapore, 2000.

[30] D. Matignon, "Stability results of fractional differential equations with applications to control processing," in Proceedings of the Institute for Mathematics and Computer Science, IEEE International Conference on Systems, pp. 963-968, Lille, France, 1996.

[31] W. Deng, "Numerical algorithm for the time fractional Fokker-Planck equation," Journal of Computational Physics, vol. 227, no. 2, pp. 1510-1522, 2007.

[32] K. Diethelm and N. J. Ford, "Analysis of fractional differential equations," Journal of Mathematical Analysis and Applications, vol. 265, no. 2, pp. 229-248, 2002. 


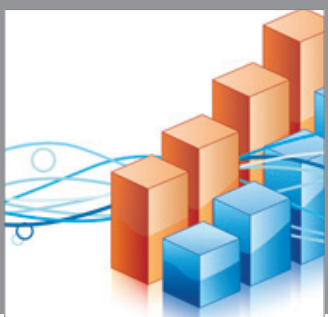

Advances in

Operations Research

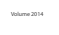

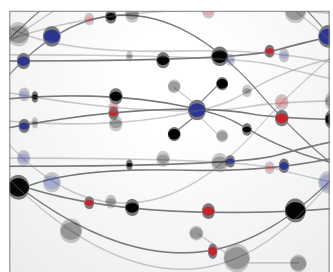

\section{The Scientific} World Journal
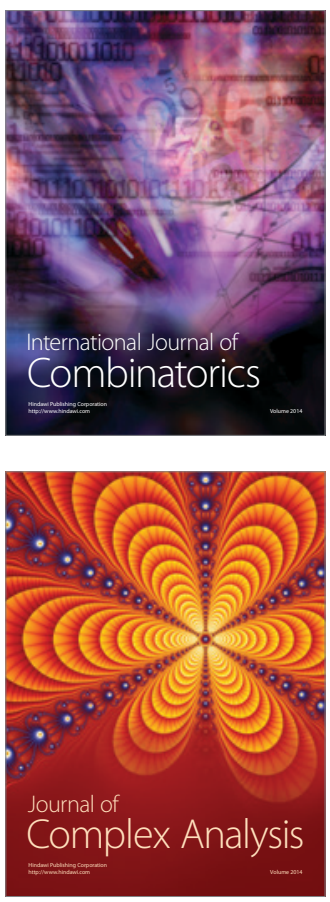

International Journal of

Mathematics and

Mathematical

Sciences
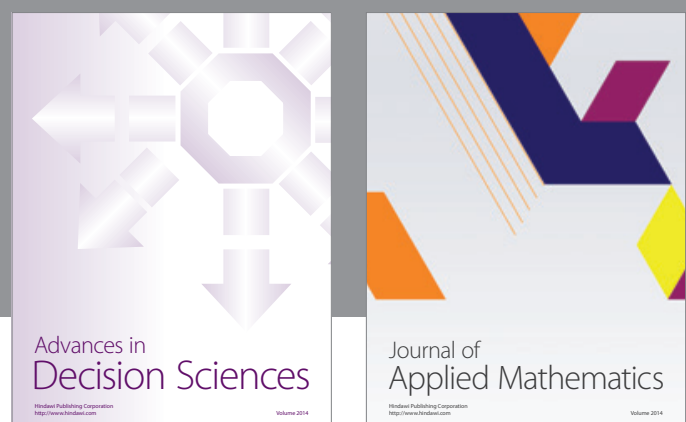

Journal of

Applied Mathematics
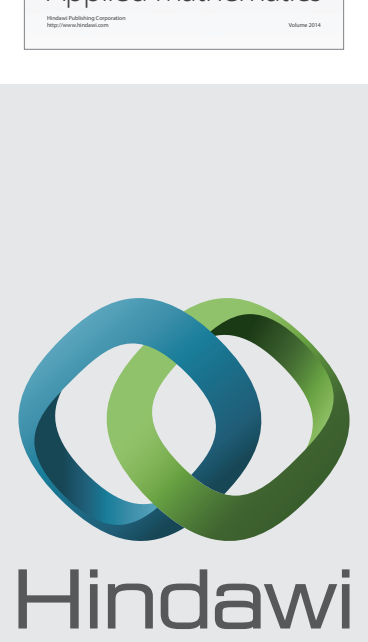

Submit your manuscripts at http://www.hindawi.com
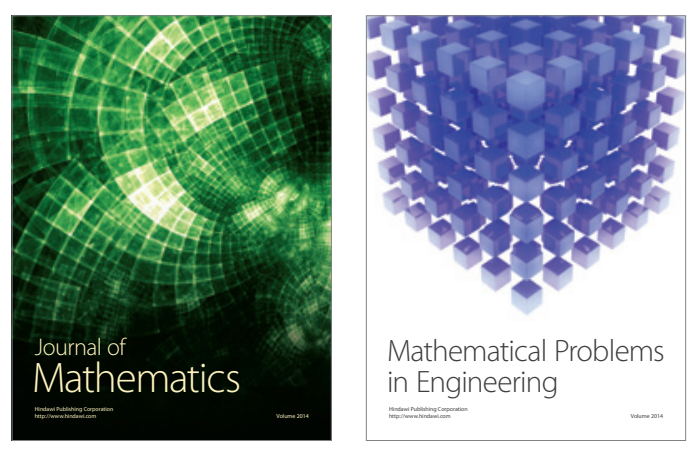

Mathematical Problems in Engineering
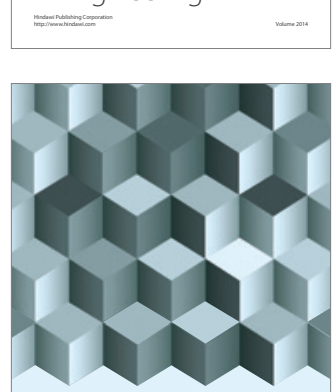

Journal of

Function Spaces
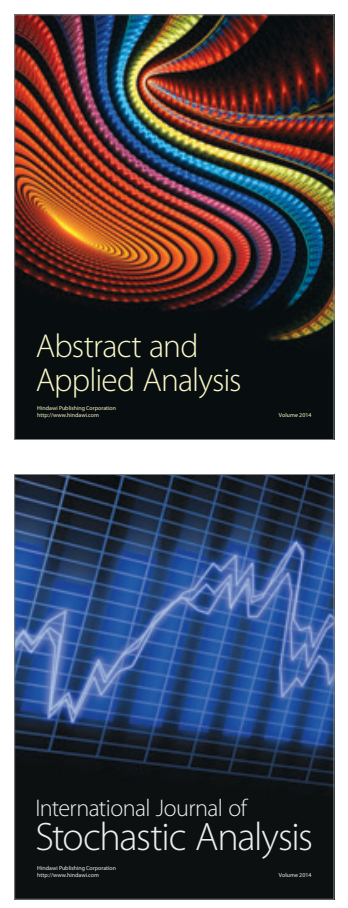

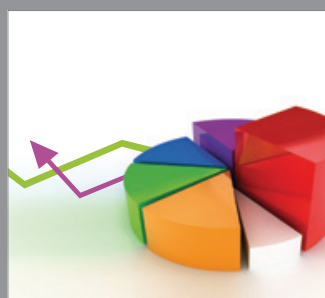

ournal of

Probability and Statistics

Promensencen
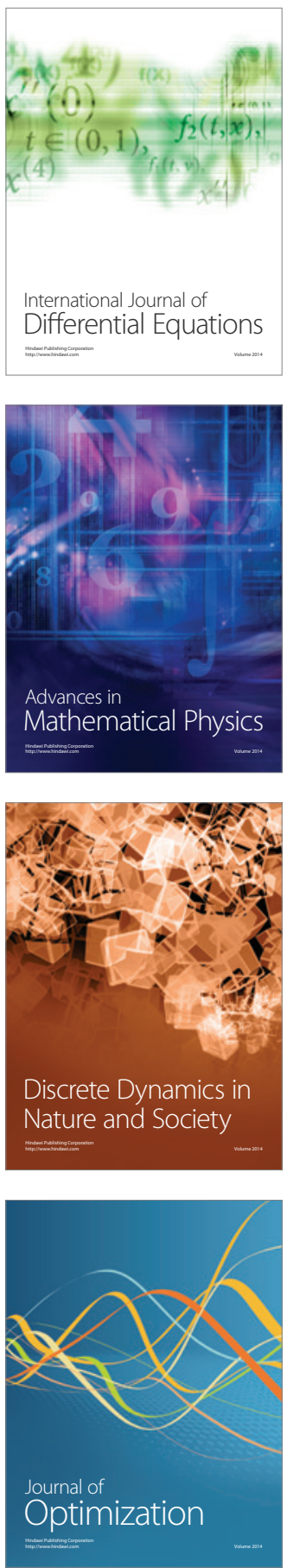